\title{
Desarrollo de comunidades forestales sustentables en Chihuahua, México
}

\author{
Concepción Luján Alvarez ${ }^{1}$ \\ Joel A. Diemer ${ }^{2}$ \\ M. Lois Stanford ${ }^{3}$
}

\begin{abstract}
RESUMEN
Esta investigación fue enfocada sobre la planeación estratégica participativa para el desarrollo sustentable de comunidades forestales en Chihuahua, México, específicamente en dos ejidos forestales localizados en la Sierra Tarahumara: Basihuare y Cusárare del municipio de Guachochi, Chih. Los objetivos fueron: 1) valorar el manejo actual de los recursos forestales en ambas comunidades, 2) conducir la metodología de la planeación estratégica participativa denominada Conferencia de Búsqueda $(C B)$ para lograr un mejor conocimiento de las necesidades de desarrollo futuro, un cambio positivo de actitud y percepciones y diseñar un plan de acción estratégico para el desarrollo sustentable de la comunidad, y 3) valorar el impacto en las actitudes y percepciones de la gente local en relación con la intervención realizada (CB) para el desarrollo de su comunidad. Los resultados mostraron que: 1) entrevistas etnográficas fueron un complemento fundamental para valorar el enfoque participativo y obtener las opiniones de los miembros de la comunidad sobre el manejo de los
\end{abstract}

recursos forestales y, 2) los residentes de Basihuare, a través de la $\mathrm{CB}$, lograron un mayor conocimiento y conciencia de las necesidades de su desarrollo futuro y se crearon mas cambios positivos en percepciones y actitudes acerca del desarrollo de su comunidad comparado con la gente de Cusárare quien no participó en este proceso de la CB. La CB ayudó a la comunidad de Basihuare a diseñar su propio plan de acción estratégico para lograr un desarrollo sustentable en el futuro y además, debido al proceso democrático y participativo que usaron, ellos llegaron a ser una comunidad planificadora. Finalmente, los resultados sugieren que el estudio puede servir como un modelo para la aplicación del enfoque de sistemas abiertos al desarrollo de comunidades forestales sustentables en la Sierra Tarahumara y en otros lugares más a nivel regional, nacional e internacional.

PALABRAS CLAVE:

Desarrollo sustentable, comunidad forestal, planeación estratégica participativa, manejo de recursos naturales.

1 Facultad de Ciencias Agrícolas y Forestales. Universidad Autónoma de Chihuahua. c.e.: clujan@smart.net.mx

2 Instituto Internacional para la Administración de Recursos Naturales, Culturales y Ambientales. New Mexico State University. E.U.A.

3 Departamento de Sociología y Antropología. New México State University. EUA.

Manuscrito recibido el 24 de septiembre de 1999. Aceptado el 9 de febrero del 2000. 


\section{ABSTRACT}

This research study focused on strategic planning for sustainable community forestry in Chihuahua State, Mexico. The research involved two forest ejidos in the Sierra Tarahumara: Basihuare and Cusarare from Guachochi Municipality. The objectives were: 1 ) assess the current forest resource management in both communities, 2) conduct a Search Conference (SC) (participative strategic planning methodology) for forest-based community development to achieve a greater awareness of development needs, positive perceptions and attitude change and to design a strategic action plan for a future community, and 3) assess the attitudinal and perceptual impact of the SC (strategic planning intervention) of local people toward community development. The results showed that: 1) ethnographic interviews were a valuable complement to and cross-check on the participatory approaches to obtaining views from the community members about their community forest resource management, and 2) the SC achieved a greater awareness of development needs and created more positive attitudes and perceptions about community development in Basihuare more than Cusárare who did not receive the intervention (SC). Additionally, the SC helped the Basihuare community to design its own strategic action plan for future development. The community not only developed its own strategic action plan, but also, because of the participative and democratic process they used, they became a planning community. Finally, the results suggest that the study could serve like a model for the application of the open system theory for the development of sustainable forestry communities in the Sierra Tarahumara and in other places more to regional, national, and international level.
KEY WORDS:

Sustainable development, community forestry, participative strategic planning, natural resource management

\section{INTRODUCCIÓN}

Muchos de los esfuerzos contemporáneos para mejorar la vida de las comunidades rurales no han llevado los beneficios a las manos de sus habitantes y simultáneamente, los recursos naturales están siendo destruídos (CIDA, 1991). Por lo tanto, la comunidad es un importante componente, aunque a menudo no es tomado en cuenta, para el manejo sustentable de los recursos naturales.

La actividad forestal en las comunidades involucra manejar dos sistemas complejos, el ecosistema forestal y el sistema social. La interacción entre estos dos sistemas determinan el éxito ó el fracaso de proyectos de desarrollo forestal (Terry, 1984). La actividad forestal en la comunidad implica un manejo colectivo para el bien común (Cabarle, 1991), por lo que para que una comunidad forestal sustentable inicie, la gente local debe estar involucrada en la toma de decisiones. Diemer y Alvarez (1995), enfatizan que los procesos participativos representan una alternativa importante para trabajar dentro del riesgo y en campos inestables para la búsqueda de un desarrollo sustentable de las comunidades forestales.

En México, el daño ecológico es extremo en varias dimensiones tales como la desforestación, desertificación, especies en extinción y contaminación del aire y el agua (Carruthers, 1996). En los últimos 50 años, la actividad forestal en México ha sentido los efectos de una política centralizada (Barton, 1991). En enero de 1992, el artículo 27 de la Constitución Mexicana fue modificado para privatizar el ejido, permitiendo a las comunidades locales organizar independientemente su vida rural (Warman, 1992). Este cambio 
sin embargo, positivo en principio, representa incertidumbre y crisis potencial para los ejidatarios (Stanford, 1994). Los gobiernos federales y estatales practican un manejo vertical sobre proyectos de desarrollo (González, 1994). Por lo tanto, el sector forestal mexicano ha mostrado serios problemas causados por las políticas impuestas por las autoridades del gobierno.

Específicamente en el Estado de Chihuahua, durante el desarrollo histórico de los aprovechamientos forestales, los bosques, típicamente, fueron aprovechados por empresas privadas y rara vez por los ejidos y comunidades; por lo tanto, ejidos y comunidades no han obtenido los mejores beneficios de sus bosques (González, 1986). La participación activa de la gente de la comunidad y el involucramiento en el proceso de toma de decisiones es uno de los aspectos fundamentales para diseñar su propio futuro deseable y alcanzable.

El éxito de las iniciativas basadas en la comunidad se apoya en que sus residentes establecen sus metas y objetivos y establecen su propio plan de acción estratégico en un marco de trabajo lógico y sistemático (Maser, 1997; Kishor y Luis, 1994). La planeación estratégica participativa provee una estructura organizacional para enfocar esos esfuerzos y creatividad en caminos productivos y de desarrollo sustentable. El proceso de planeación estratégica participativa a través de la metodología de la Conferencia de Búsqueda (CB) responde tres preguntas básicas: ¿Cuál es la interrelación que existe entre el ambiente externo y la comunidad para su desarrollo? ¿Dónde está nuestra comunidad ahora? ¿Cómo queremos que nuestra comunidad sea en el futuro? y ¿Cómo podemos nosotros llegar allí mas efectivamente?

\section{OBJETIVOS}

Este estudio se enfocó sobre la "Planeación Estratégica Participativa para el Desarrollo Sustentable de Comunidades Forestales en Chihuahua, México". Los objetivos de la investigación fueron: 1) valorar el manejo actual de los recursos forestales en ambas comunidades para identificar el patrón de manejo de los mismos y apoyar el análisis de actitudes y percepciones de la gente local en relación con el desarrollo forestal comunitario, 2) conducir la metodología de la Conferencia de Búsqueda (CB) para realizar una planeación estratégica participativa para el desarrollo sustentable de la comunidad de manera de lograr un mayor conocimiento de las necesidades de desarrollo, un cambio positivo de actitud y percepciones y diseñar un plan de acción estratégico para su desarrollo sustentable en el futuro $y, 3)$ valorar el impacto actitudinal y de percepciones causado por la aplicación de la CB en la gente local en relación con el desarrollo sustentable de la comunidad y el manejo de sus recursos forestales.

\section{METODOLOGÍA}

El estudio involucró dos ejidos forestales en la Sierra Tarahumara: Basihuare y Cusárare en el municipio de Guachochi en Chihuahua, Mexico. Las etapas de la investigación para el logro de los objetivos planteados consistieron en las siguientes:

1. Investigación cualitativa para valorar el manejo de los recursos forestales en ambas comunidades. Esta fase de la investigación fue conducida a través del uso de los métodos de entrevistas etnográficas y observación directa. Para ello, fue necesario primeramente realizar una selección de informantes claves en ambas comunidades, con el criterio de que fueran personas locales que tuvieran uin profundo conocimiento y experiencia acerca de la vida de la 
comunidad y de la actividad forestal. El método de bola de nieve (snowball sampling) (Bernard, 1994) fue utilizado para hacer dicha selección. Posteriormente, una entrevista etnográfica fue aplicada a los informantes claves, seleccionados en ambas comunidades. La entrevista incluyó 15 preguntas relacionadas con los siguientes aspectos: formas de aprovechamiento de los bosques, planeación de su manejo forestal, formas de participación de la gente local en el manejo de sus recursos naturales, capacitación y entrenamiento de la gente local, mercado de productos forestales y beneficios recibidos por la gente local como resultado de la actividad forestal. Específicamente, algunos ejemplos de preguntas integradas al cuestionario para realizar la entrevista etnográfica son los siguientes:

- ¿El ejido ha manejado bien sus recursos forestales?

Si-- No-Por qué?

- ¿Cuales son los principales factores que han afectado los recursos forestales del ejido? Cuál es el más crítico? Cómo se podría solucionar este problema en el futuro?

- ¿Cómo afecta el tipo de tenencia de la tierra el manejo y conservación de los recursos forestales del ejido?

- ¿Cómo ha planeado el ejido el desarrollo de sus actividades forestales tanto en el pasado como en el presente?

- ¿Cómo la gente del ejido ha participado en el diseño e implementación de proyectos de desarrollo para al manejo de su recursos forestales?

2. Diseño de la investigación para hacer el estudio comparativo actitudinal y de percepciones como resultado de la conducción de la $\mathrm{CB}$ en Basihuare. Estas comunidades, antes mencionadas, fueron selecciondas para realizar un análisis comparativo de las actitudes y percepciones de la gente local respecto a su participación en el diseño de planes de acción estratégico para el desarrollo sustentable de la comunidad forestal. El análisis evaluó los efectos e impacto de la intervención a través de la conducción de una Conferencia de Búsqueda en la comunidad de Basihuare comparándola con la comunidad de Cusárare la cual no recibió el tratamiento.

El diseño de investigación utilizado en este estudio fue el denominado "Preprueba-postprueba y grupo de control" (Bernard, 1994 (Fig. 1). El estudio consistió primeramente en la aplicación de una pre-evaluación (cuestionario para análisis de actitudes y percepciones) en ambas comunidades (Fig. 1). El cuestionario consistió de 20 declaraciones incluyendo tópicos sobre la participación de la gente local y el diseño de planes de acción estratégicos para el desarrollo sustentable de la comunidad forestal. El cuestionario fue diseñado usando la Escala de Likert para medir las actitudes y percepciones. Algunos ejemplos de estas declaraciones usando la Escala de Likert (fuertemente de acuerdo, de acuerdo, incierto 0 no sé, en desacuerdo, fuertemente en desacuerdo) para cada una de ellas son las siguientes:

- El programa de manejo forestal actual contribuye a la buena administración de los recursos naturales para el desarrollo futuro de la comunidad.

- La gente local tiene poco o nada de interés en participar en la planeación e implementación del manejo de sus recursos forestales.

- Instituciones externas a la comunidad son responsables de la elaboración de los programas de 
manejo forestal de la misma comunidad.

- Se pueden obtener mejores resultados en la planeación e implementación de los programas de desarrollo forestal en la comunidad a través de intereses aislados y personales

- La gente de la comunidad está satisfecha con los beneficios que obtiene del aprovechamiento de sus recursos forestales.
Después de la pre-evaluación, una Conferencia de Búsqueda (intervención) (Fig. 2) (Emery y Purser, 1996) fue conducida en la comunidad de Basihuare. Mas tarde, una post-evaluación (usando el mismo cuestionario que en la preevaluación) fue aplicada en ambas comunidades como se mostró anteriormente en la figura 1.

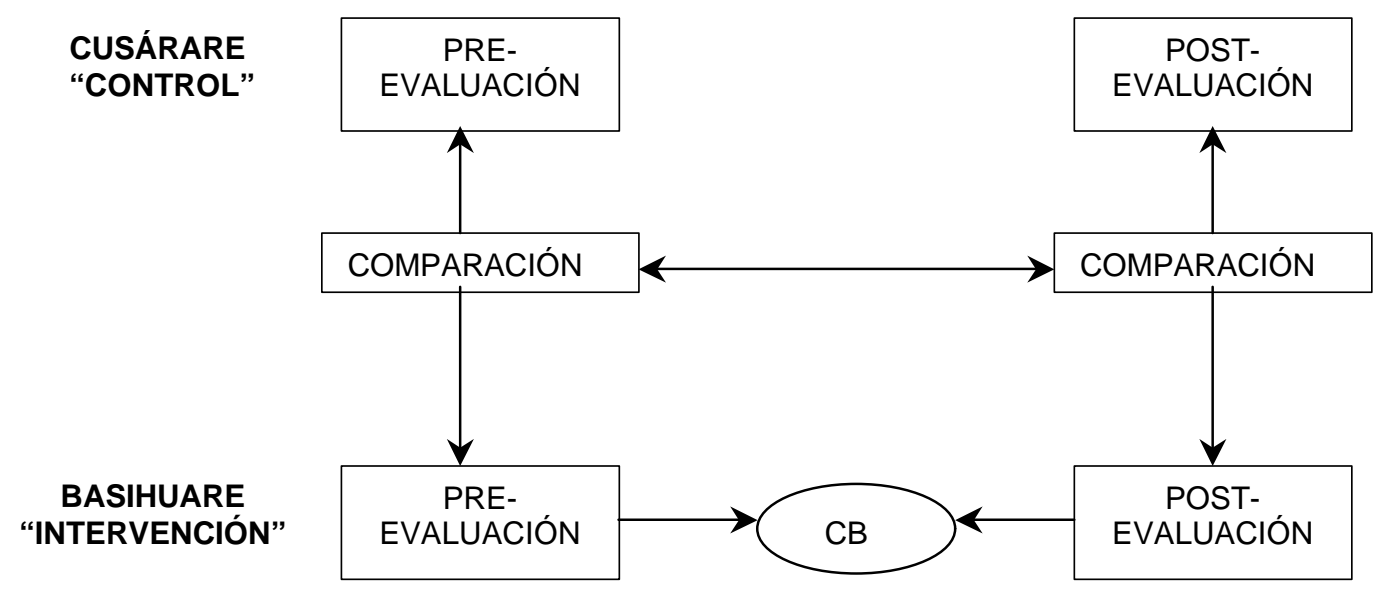

$\mathrm{CB}=$ Conferencia de Búsqueda (Intervención para la Planeación Estratéaica Participativa

Figura 1. Diseño: "Preprueba-postprueba y grupo de control

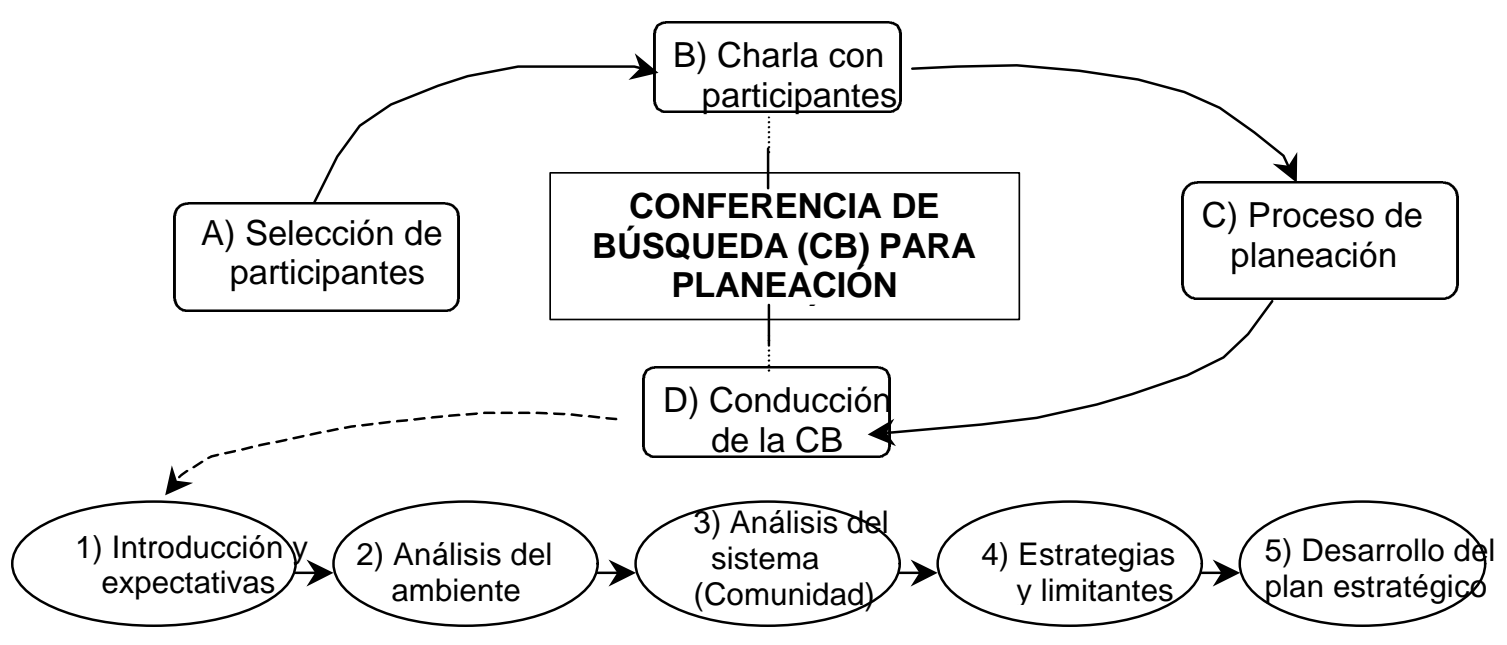

Figura 2. Proceso de la Conferencia de Búsqueda para la Planeación Estratégica Participativa 
Adicionalmente, una investigación cualitativa, incluyendo entrevistas etnográficas, observación de participantes y reuniones de seguimiento, fue realizada para obtener mayor información complementaria para la evaluación del manejo de los recursos forestales y el impacto de la intervención a través de la conducción de la Conferencia de Búsqueda. Comparaciones fueron llevadas a cabo entre las dos comunidades antes y después de la conducción de la CB para medir los cambios en actitudes y percepciones como un efecto de la intervención.

3. Valoración del impacto actitudinal y de percepciones como efecto de la intervención-CB. Los datos resultantes de la pre-evaluación y de la postevaluación en ambas comunidades fueron analizados estadísticamente usando el método "General Linear Model" (GLM) por medio del paquete SAS. El análisis fue estructurado para probar los principales efectos entre la comunidad intervención (Basihuare) y la control (Cusárare) incluyendo las interacciones. El objetivo del análisis fue examinar los efectos en ambas comunidades considerando el tiempo antes y después de la conducción de la $\mathrm{CB}$ en Basihuare.

\section{RESULTADOS}

Los resultados mostraron que: 1) las entrevistas etnográficas fueron un importante complemento, sobre el enfoque participativo, en la obtención de puntos de vista y opiniones de los miembros de la comunidad acerca del manejo de sus recursos forestales. Basados en la caracterización del manejo de los recursos forestales, los principales problemas percibidos por la gente de ambas comunidades son mostrados en la tabla 1 y 2) a través de la conducción de la $\mathrm{CB}$, la gente local de la comunidad de Basihuare (intervención) logró un mayor entendimiento y conocimiento de las necesidades de desarrollo de su comunidad y creó mas actitudes y percepciones positivas respecto al desarrollo de la comunidad. Como resultado, es posible notar que la $\mathrm{CB}$ impactó positivamente en las percepciones y actitudes de los participantes en relación a su participación para el desarrollo sustentable de su comunidad en el futuro. Estadísticamente, hubo diferencias significativas en cambios de actitudes y percepciones en la gente que participó en la CB (Basihuare) comparada con la gente que no lo hizo (Cusárare) (Fig. 3).

Adicionalmente, en la tabla 2 se muestran dos ejemplos específicos de las declaraciones sobre actitudes y percepciones incluídas en el cuestionario las cuales fueron evaluadas en la pre y post-evaluación en ambas comunidades. Se presenta el procedimiento de análisis de las declaraciones contenidas en el cuestionario. Se observa el comportamiento del puntaje promedio, el nivel de significancia y el efecto obtenido en los participantes en ambas comunidades antes y después de la CB de acuerdo a la Escala de Likert.

En ambas declaraciones se muestra una diferencia significativa entre los puntajes promedio de la pre y postevaluación en la comunidad de Basihuare (intervenida) con un $\alpha=0.05$. En contraste, en la comunidad de Cusárare (control) no hubo diferencia significativa en estas declaraciones entre la pre y postevaluación al mismo nivel de $\alpha$. Por lo tanto, estos resultados muestran el impacto que tuvo la conducción de la CB.

En el análisis hecho para todas las declaraciones contenidas en el cuestionario, en la comunidad intervenida (Basihuare), los puntajes promedio mostraron un incremento y diferencia significativa $(\alpha=0.05)$ entre la pre y postevaluación después de la aplicación 
Tabla 1. Principales problemas identificados por los informantes claves (IC) acerca del manejo de los recursos forestales en ambas comunidades

\begin{tabular}{||l|c|c||}
\hline \multicolumn{1}{|c|}{ PROBLEMAS } & BASIHUARE & \multicolumn{2}{c|}{ CUSÁRARE } \\
\hline Sobre-explotación del bosque & Si $70 \%$ IC & Si $100 \%$ IC \\
\hline Corrupción & Si $90 \%$ & Si $100 \%$ \\
\hline $\begin{array}{l}\text { Altos costos de prestadores de servicios técnicos } \\
\text { forestales }\end{array}$ & Si $80 \%$ & Si $90 \%$ \\
\hline $\begin{array}{l}\text { Falta de recursos financieros e inversiones para el } \\
\text { manejo forestal. }\end{array}$ & Si $100 \%$ & Si $100 \%$ \\
\hline $\begin{array}{l}\text { Falta de planeación e involucramiento de la gente } \\
\text { local para el manejo forestal }\end{array}$ & Si $100 \%$ & Si $100 \%$ \\
\hline Falta de ganancias por actividad del aserradero & Si $100 \%$ & Si $100 \%$ \\
\hline
\end{tabular}

Tabla 2: Ejemplos de declaraciones de actitudes y percepciones y sus puntajes promedios en ambas comunidades.

CONTROL (Cusárare)

INTERVENCION (Basihuare)

\begin{tabular}{|c|c|c|c|c|c|c|}
\hline DECLARACION & $\begin{array}{c}\text { PRE- } \\
\text { EVALUACIÓN } \\
\text { Puntaje } \\
\end{array}$ & $\begin{array}{c}\text { POST- } \\
\text { EVALUACIÓN } \\
\text { Puntaje } \\
\end{array}$ & $P>|t|$ & $\begin{array}{c}\text { PRE- } \\
\text { EVALUACIÓN } \\
\text { Puntaje } \\
\end{array}$ & $\begin{array}{c}\text { POST- } \\
\text { EVALUACIÓN } \\
\text { Puntaje } \\
\end{array}$ & $P>|t|$ \\
\hline $\begin{array}{l}\text { Es necesario } \\
\text { definir un plan } \\
\text { de trabajo de la } \\
\text { comunidad para } \\
\text { un mejor } \\
\text { manejo de los } \\
\text { recursos } \\
\text { naturales }\end{array}$ & 2.20 & 2.40 & 0.5103 & 2.26 & 4.26 & 0.0001 \\
\hline $\begin{array}{l}\text { Tu participación } \\
\text { en la planeación } \\
\text { de actividades } \\
\text { para el } \\
\text { desarrollo de la } \\
\text { comunidad con } \\
\text { otras gentes de } \\
\text { la misma } \\
\text { comunidad les } \\
\text { permite lograr un } \\
\text { mejor desarrollo } \\
\text { y beneficios en } \\
\text { el futuro }\end{array}$ & 3.13 & 2.80 & 0.2905 & 3.20 & 4.01 & 0.0062 \\
\hline $\begin{array}{l}\text { Nota*: } \\
1=\text { Fuertemente en } \mathrm{d} \\
2=\text { Desacuerdo }\end{array}$ & & & $\begin{array}{l}3=\mathrm{Nc} \\
4=\mathrm{De} \\
5=\mathrm{Fu}\end{array}$ & $\begin{array}{l}\text { decidido } \\
\text { acuerdo } \\
\text { ertemente de acue }\end{array}$ & & \\
\hline
\end{tabular}




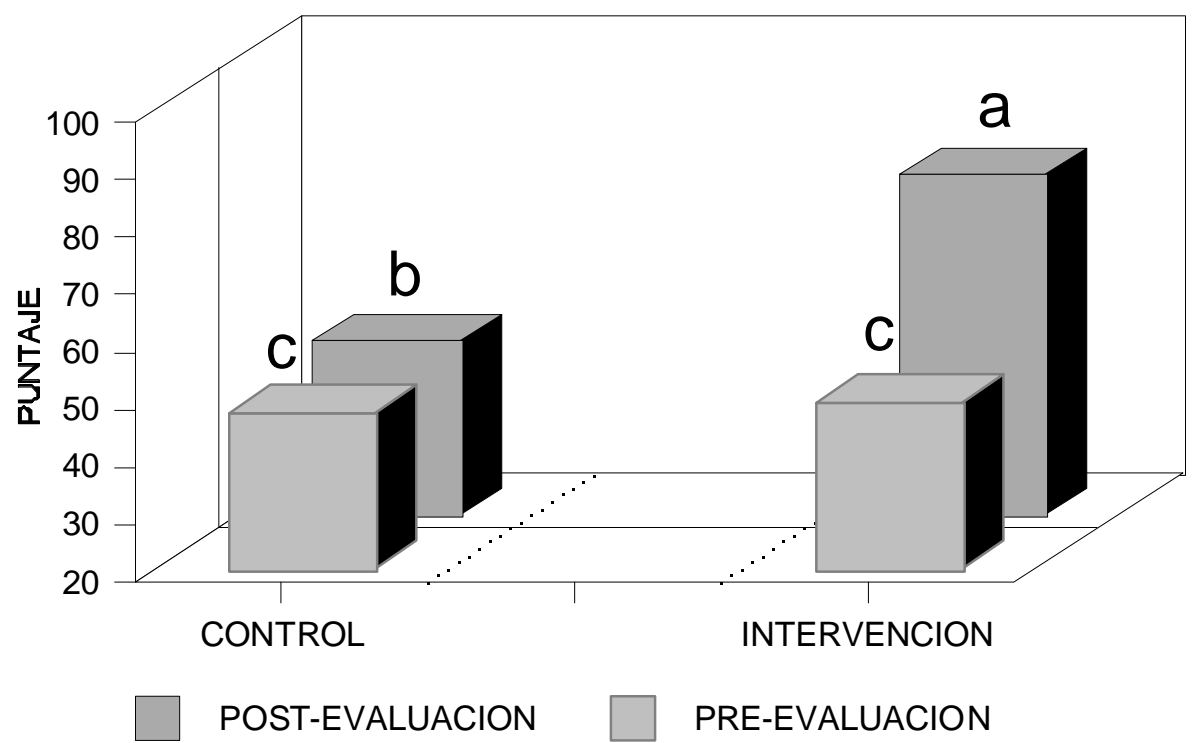

Nota: Tratamientos con diferentes letras son significativamente diferentes, alpha $=0.05$

Figura 3. Comparaciones en las comunidades: el efecto de la intervención (CB).

de la CB; sin embargo, en la comunidad control (Cusárare), se observó que no hubo diferencia significativa $(\alpha=0.05)$ entre la pre y post-evaluación en las declaraciones y los puntajes promedio fueron similares.

Adicionalmente, la CB ayudó a la comunidad de Basihuare a diseñar su propio plan de acción estratégico para su desarrollo sustentable en el futuro; por lo que la comunidad llegó ser una comunidad planificadora. Las metas estratégicas establecidas por la comunidad fueron:

1. Crear empleos a través de la construcción y operación de cabañas turísticas y tiendas de artesanías.

2. Incrementar la producción agrícola y ganadera de la comunidad.
3. Mejorar las condiciones tecnológicas del aserradero del ejido, e instalar la energía eléctrica y el agua potable para la comunidad.

4. Mejorar la educación y los servicios médicos.

5. Erradicar los problemas de alcohol y drogas.

6. Obtener el apoyo de las autoridades superiores para el desarrollo de diferentes actividades para beneficio de la comunidad.

Lo anterior significa que el crear empleos por medio de la construcción y operación de cabañas turísticas estaría implicando el uso de paisaje del bosque más no el uso y deterioro físico de los recursos forestales. Además, cuando diseñan, fabrican y venden artesanías, se 
proporciona un valor agregado a la materia prima (madera), además de la producción de la producción de madera aserrada. También, decidieron aumentar y mejorar la agricultura y producción del ganado usando para ello los terrenos de uso agrícola existentes actualmente y de esta manera evitar perturbar los recursos forestales, la fauna y otros recursos asociados. Por lo tanto, la comunidad busca ser más eficiente en la actividad agrícola y ganadera reduciendo la presión sobre los recursos forestales. Además, decidieron mejorar el funcionamiento y niveles de productividad del aserradero (sin seguir afectando al bosque intensivamente), así como la calidad de productos, mientras se reducen los costos de operación y administrativos, obteniendo de esta manera mayores ingresos para beneficio de la comunidad.

Si la comunidad tiene los recursos necesarios para llevar a cabo la puesta en práctica del plan de acción estratégico generado por ellos mismos de acuerdo a sus necesidades e intereses, la comunidad podrá obtener mayores ingresos para mejorar su alimentación, así como los servicios de educación, salud, agua potable, entre otros. Por consiguiente, estas acciones reforzarán el esfuerzo por alcanzar en el futuro el desarrollo sustentable de la comunidad, ya que estarían al mismo tiempo protegiendo y recuperando sus recursos forestales y trabajando con otros recursos asociados sin destruir el bosque para lograr mejor calidad de vida. Con el establecimiento de estas metas estratégicas y el diseño del plan de acción estratégico, los miembros de la comunidad han tomado un paso importante hacia mejorar las condiciones socioeconómicas y ecológicas, que beneficien a la comunidad, sus recursos y su ambiente.

Adicionalmente, el establecimiento de una comunicación directa entre la comunidad y entidades externas ayudaron a identificar estrategias adicionales y a conformar sus proyectos técnicos y financieros para apoyar a la comunidad en su desarrollo futuro. Debido a que estas entidades externas pueden ser muy receptivas para ayudar a la comunidad, Basihuare tiene una oportunidad valiosa para trabajar con ellas ejecutar su plan de acción estratégico.

\section{DISCUSIÓN}

Los resultados obtenidos en la investigación sobre los cambios positivos en valores y percepciones en los participantes son fundamentalmente importantes para el desarrollo sustentable de la comunidad de Basihuare. Estos resultados obtenidos no apoyan los resultados presentados en otros estudios hechos por investigadores tales como Albrecht y Geertsen (1982), quienes sugieren que los residentes en comunidades con niveles de pobreza cada vez mayores, necesariamente tienen una actitud negativa para su desarrollo, por lo que esto contribuye aún mas a la pobreza en la comunidad. En contraste, los resultados logrados en el presente estudio, después de la conducción de la CB para el desarrollo de la comunidad, acuerdan con Mallik y Rahman (1994), quienes dicen que la gente local son los mas interesados en el bienestar y estabilidad económica de sus comunidades. Es necesario que su conocimiento sea considerado en el diseño e instrumentación de proyectos para el desarrollo sustentable de la comunidad forestal. En el presente estudio, aun cuando Basihuare tiene escasez de recursos financieros para inversiones, falta de ganancias en el aserradero y falta de planeación de recursos, la gente no mostró actitudes negativas para el desarrollo de la comunidad en el futuro. En el momento en que los recursos estén disponibles, ellos están motivados e interesados para llevar a cabo la puesta en marcha de los planes de acción estratégicos para el desarrollo sustentable de la comunidad. 
En resumen, la comunidad de Basihuare, estableció prioridades en la definición de sus objetivos/metas estratégicas y el plan de acción estratégico de acuerdo con sus necesidades e intereses y que sustenta importantes criterios para buscar una transición hacia el desarrollo sustentable. Lo anterior, es debido a que la priorización y el diseño final del plan estratégico estuvo basado en la participación e involucramiento de comunidad en todo el proceso de planeación democrática participativa. Además, el plan inlcuye en primera instancia y de acuerdo a la priorización de metas estratégicas, la necesidad de crear empleos para la gente local $y$, consecuentemente, mejorar otras actividades económicas (artesanías, agricultura y ganadería) y condiciones sociales (servicios de salud, alimentación, vivienda y vestido) y al mismo tiempo proteger y recuperar los recursos forestales deteriorados.

Con el propósito de implantar este plan de acción, la misma comunidad decidió solicitar asesoría técnica a varias instituciones tales como la Universidad Autónoma de Chihuahua, New Mexico State University, Gobierno del EstadoCoordinación Estatal de la Tarahumara, entre otras, con el objeto de elaborar los proyectos técnicos y financieros específicos de acuerdo a la naturaleza del contenido del plan de acción. Es importante mencionar que actualmente la comunidad se encuentra en esta etapa de avance de apoyo técnico, para enseguida presentar los proyectos técnicos $y$ financieros detallados a diferentes instancias financieras, tanto en México como en Estados Unidos y Canadá y lograr la obtención de los recursos económicos que permitan a dicha comunidad la ejecución de su plan de acción estratégico para su desarrollo futuro.

\section{CONCLUSIONES}

En conclusión, el estudio ha intentado examinar los efectos de la CB sobre los valores y percepciones de los residentes locales con respecto al desarrollo futuro de su comunidad. La gente local reconoce los problemas que han tenido en el manejo de sus recuros forestales. Los resultados confirmaron la hipótesis planteada inicialmente. También, los resultados sugieren que el estudio puede servir de modelo para la aplicación de la Teoría de Sistemas Abiertos a través de la conducción de Conferencias de Búsquedas con un enfoque hacia el desarrollo sustentable de las comunidades forestales en la Sierra Tarahumara y otros lugares tanto en México como fuera de él.

Uno de los aspectos importantes que puede permitir la instrumentación de este modelo de desarrollo participativo en diferentes condiciones socioculturales, económicas y ecológicas, es la versatilidad, flexibilidad y dinámica en el sistema, así como la visión holística que presenta la naturaleza y el proceso de conducción de la Conferencia de Búsqueda, como una metodología para la planeación estratégica participativa y democrática.

Adicionalmente, esta experiencia presenta elementos importantes debido a los cuales los dueños de los recursos forestales deben de ser los principales actores en el proceso de toma de decisiones para su desarrollo futuro, toda vez que son ellos los que viven con las consecuencias de cualquier acción que se tome en su comunidad. Otras razones adicionales para que la gente local sea el principal actor son: su conocimiento representa toda una historia de experiencias acumuladas sobre como la comunidad ha manejado sus recursos, aún en condiciones adversas y la gente local tiene una racionalidad ecológica la cual representa un componente decisivo en la oeganización de sus estrategias de vida. 


\section{RECONOCIMIENTOS}

Este estudio fue ganador del PREMIO AL MERITO NACIONAL FORESTAL 1998 otorgado por el Gobierno Federal a través de la Secretaría de Medio Ambiente, Recursos Naturales y Pesca (SEMARNAP).

\section{REFERENCIAS}

Albrecht, D.E. y H.R. Geertren. 1982. Population growth in rural communities and residents' perceptions of its consequences. Journal of the Community Development Society 13(2): 75-90.

Barton, D. 1993. The struggle for the forest: conservation and development in the Sierra de Juárez. Grassroots Development Vol. 15/3: 13-25.

Bernard, H.R. 1994. Research methodology in Anthropology: qualitative and quantitative approaches. Sage Publications. 585 p.

Cabarle, B.1991.Community Forestry and the Social Ecology of Development. Grassroots Development 15 (3):3-9.

Carruthers, D. 1996. Indigenous ecology and the politics of linkage in Mexican social movements. Third World Quarterly 17(5): 1007-1028.

CIDA (Canadian International Development Agency). 1991. Sustainable development: framework paper. $19 \mathrm{p}$.

Diemer, J. y R. Alvarez. 1995. Sustainable community, sustainable forestry: a participatory model. Journal of Forestry 93(11): 10-14.

Emery, M. y Ronald E.P. 1996. The search conference: a powerful method for planning organizational change and community action. Jossey-Bass. CA. EUA. 320p.

González, H.J. 1986. Algunas perspectivas de la actividad forestal en el Estado de Chihuahua. Tesis de Licenciatura. Chapingo, Mex. División de Ciencias Forestales. Universidad Autónoma de Chapingo. $134 \mathrm{p}$.

González, H.J. 1994. Estrategia para orientar el rumbo de los aprovechamientos forestales en México. Consultoría Forestal Tutuaca, S.A. de C.V. Chihuahua, Chih. 29 p.

Kishor, N. y C. Luis. 1994. Sustainable forestry: can it compete? Finance and Development 34/4:36-39.

Mallik, A. y H. Rahman. 1994. Community forestry in developed and developing countries: a comparative study. The Forestry Chronicle 70(6): 731-735.

Maser, Ch. 1997. Sustainable community development: principles and concepts. St Lucie Press. Delray Beach, Florida. 257p.

Stanford, L. 1994. The privatization of Mexico's ejidal sector: examining local impacts, strategies, and ideologies. Urban Anthropology. 23 (2)-3: 98-119.

Terry, R.A. 1984. Community forestry-the social view. In: Community Forestry: some aspects. United Nations Development Programme, Environment and Policy Institute and Food and Agricultural Organization. 39-46.

Warman, A. 1992. Notas para la presentación del tema: el destino del campesinado mexicano. Documento presentado en la conferencia "Las transformaciones de la agricultura mexicana: oportunidades, dilemas e implicaciones. Univ. of California. Berkeley, CA. Dic. 3-4, 1992. 